\title{
Percutaneous Tracheostomy in Patients at High Risk of Bleeding Complications: A Retrospective Single-center Experience
}

\author{
Sachin P Sasane ${ }^{1}$, Madhavi M Telang ${ }^{2}$, Zeyad F Alrais ${ }^{3}$, Ali HNS Alrahma ${ }^{4}$, Khalid I Khatib ${ }^{5}$
}

\begin{abstract}
Aims: To study the bleeding complications in patients undergoing percutaneous tracheostomy and who are at high risk of these complications (due to thrombocytopenia, use of anticoagulant or antiplatelet agents, and difficult anatomy).

Materials and methods: A retrospective study was undertaken, which included all patients undergoing percutaneous tracheostomy in the medical intensive care unit (MICU) of Rashid Hospital, Dubai, over a period of 15 months. Percutaneous tracheostomy was performed by senior medical intensivists using the single-tapered dilator technique under fiber optic bronchoscopic guidance. All patients underwent ultrasonographic evaluation of the neck to look for difficult anatomy and to determine the size of tracheostomy tube, etc. Patients were divided into two groups, those who were deemed to be at high risk of bleeding complications were compared with patients without any risk factors for bleeding complications. Other complications such as pneumothorax and tracheal leak were also looked for and were documented, if present. The data were summarized using descriptive statistics and the Fischer's exact test of significance was used for frequency distribution cross tables, at $5 \%$ level of significance ( $p$ value cutoff $<0.05$ ).

Results: One hundred and fifty-nine patients underwent percutaneous tracheostomy during the period of study. The age-group of patients ranged from 21 years to 104 years and males were predominant $(65.41 \%)$. Of the $87(54.71 \%)$ patients with one or more risk factors for bleeding, $53(60.92 \%)$ patients had at least one risk factor for bleeding complications, while $34(39.08 \%)$ had more than one risk factors. Bleeding was seen in total of two patients out of which one patient was in the group at risk of bleeding complications.

Conclusion: Percutaneous tracheostomy is a relatively safe procedure with very low rate of complications when performed with due precautions. Even in patients deemed to be at high risk of complications, the rate of complications is very low.

Keywords: Bleeding complications, Intensive care, Percutaneous dilatational tracheostomy.

Indian Journal of Critical Care Medicine (2020): 10.5005/jp-journals-10071-23341
\end{abstract}

\section{INTRODUCTION}

Percutaneous dilatational tracheostomy (PDT) is one of the most common procedures performed in the ICU, along with central venous catheterization. It is preferred in critically ill patients as compared to surgical tracheostomy (ST) due to lesser wound infections, fewer episodes of bleeding, cost savings, lesser decision to procedure time, and no need to transfer patients to the operation theater. ${ }^{1-3}$ The most common indications of a tracheostomy in ICU patients are prolonged mechanical ventilation (MV) to facilitate weaning and airway protection and occasionally to facilitate pulmonary toilet. ${ }^{4}$ While there are various techniques for performing PDT, the single-step dilational technique (SSDT) has become the most preferred method for performing the procedure. ${ }^{5-7}$ There are few contraindications for PDT, the chief among them are local infections, coagulopathy, difficult anatomy (morbid obesity, short neck, anterior cervical spine fracture/dislocation, aberrant blood vessels, abnormal thyroid placement, etc.), and severe gas exchange abnormalities. ${ }^{2,4}$ Even these contraindications are not absolute but relative. Studies have shown that PDT can be safely performed in patients with severe thrombocytopenia and those requiring very high oxygen. ${ }^{8,9}$ Also some of the anatomical variations can be confirmed by performing a ultrasound (USG) of the neck prior to the tracheostomy procedure, so as to prevent anatomical surprises during the procedure. ${ }^{10-12}$ The rate of complications is low in patients undergoing PDT and complications are related to local bleeding and stomal infections. Risk factors for bleeding are

\footnotetext{
${ }^{1-4}$ Department of Medical Intensive Care Unit, Rashid Hospital, Dubai, United Arab Emirates

${ }^{5}$ Department of Medicine, Smt. Kashibai Navale Medical College, Pune, Maharashtra, India
}

Corresponding Author: Khalid I Khatib, Department of Medicine, Smt. Kashibai Navale Medical College, Pune, Maharashtra, India, Phone: +91-20-25439140, e-mail: drkhatibkhalid@gmail.com

How to cite this article: Sasane SP, Telang MM, Alrais ZF, Alrahma AHNS, Khatib KI. Percutaneous Tracheostomy in Patients at High Risk of Bleeding Complications: A Retrospective Single-center Experience. Indian J Crit Care Med 2020;24(2):90-94.

Source of support: Nil

Conflict of interest: None

presence of coagulation abnormalities and abnormal anatomy, but the precise coagulation abnormalities that are responsible for bleeding are not clear. There is conflicting evidence regarding relationship between severe thrombocytopenia and heparin treatment, and the episodes of bleeding complications and best practice guidelines are not there. ${ }^{8,13}$ According to a study in German patients, PDT done by experienced staff with bronchoscopy support did not increase the risk of bleeding in patients with severe thrombocytopenia (platelet count of $<50,000$ ), but the presence of heparin infusion did increase the risk. ${ }^{8}$ Another study, also on German patients who underwent PDT done by experienced staff, demonstrated increased risk of bleeding in patients with severe 
thrombocytopenia (platelet count of $<50,000$ ), abnormal activated partial thromboplastin time (aPTT) ( $>50$ seconds), and presence of more than one coagulation abnormalities, but not with low-dose heparin infusion. ${ }^{13}$ The American Society of Anesthesiologists taskforce for major surgery recommends platelet transfusion for severe thrombocytopenia and fresh frozen plasma for abnormally prolonged aPTT/prothrombin time prior to major surgery. ${ }^{14}$

We decided to conduct a retrospective study to understand the practice of PDT in our ICU especially with regard to bleeding complications and in patients at risk of these complications.

\section{Materials and Methods}

A retrospective study was undertaken in the 48-bedded adult MICU of Rashid Hospital, Dubai (RHD), after Ethical Committee approval. The need for consent was waived as it was a retrospective review of hospital electronic medical records. All patients who underwent PDT from October 1, 2017, to December 31, 2018, were included in the study. The MICU at RHD is a 48-bedded mixed medicalsurgical unit performing approximately 100 PDTs per year. The MICU is staffed with 26 medical intensivists who provide $24 \times 7$ cover on all days of the week. Of the 26 medical intensivists, 2 are consultants ( $>20$ years' experience) and 13 are specialist senior registrar ( $>15$ years' experience), while 11 are specialist registrar ( $>10$ years' experience). Each duty has at least three specialist senior registrars/specialist registrars with the consultant intensivist present for 8 hours a day during the daytime and he/she is also available on-call in an emergency. Decision for tracheostomy is taken by the consultant intensivist during the ICU rounds. The decision is then conveyed to the patient and/or patient relative and consent for tracheostomy is taken. Once it is decided to go ahead with the tracheostomy, the patient is evaluated, examined, and USG of the neck region is carried out by the on-duty specialist senior registrar for determining the neck anatomy (tracheal rings, thyroid gland, and adjoining blood vessels) and decision for suitability to perform PDT is taken. All PDTs are performed by specialist senior registrar/specialist registrar (>50 PDTs done independently) along with consultant intensivist (>100 PDTs done independently) at the bedside. All patients were more than 18 years of age and each received intravenous general anesthesia with neuromuscular blockade. Each procedure was undertaken with fiber optic bronchoscopic guidance to ensure correct placement of the guidewire. The single-tapered dilator technique of PDT, as described previously, is the standard technique of tracheostomy in our ICU. ${ }^{15}$

\section{Data Collection}

Data collected from hospital electronic medical records (EMR) were demographic and perioperative data of each patient, which included age, gender, reason for admission, number of days of ICU admission, and intubation prior to tracheostomy, indication for tracheostomy (prolonged MV and to facilitate weaning, airway protection, pulmonary toileting), intraoperative and postoperative complications, and presence of risk factors for bleeding (treatment with antiplatelet agents or anticoagulants, difficult anatomy, platelet count to determine presence of and level of thrombocytopenia, and coagulogram).

A complication was said to have occurred if (a) oxygen saturation fell below $88 \%$ during the procedure, (b) hypotension or cardiac arrest occurred, (c) multiple (>3) attempts were needed for tracheal cannulation, (d) bleeding was present, which required more than three swabs to be used and/or requiring surgical intervention, (e) endotracheal tube (ETT) cuff puncture, (f) tracheal rings were fractured or posterior tracheal wall was injured and visualized on bronchoscopy, (g) presence of subcutaneous surgical emphysema, (h) pneumothorax, (i) tracheostomy tube was misplaced (false passage, accidental extubation), and (j) PDT had to be converted to ST.

Patients were defined as high risk of bleeding complications if they had any of the following factors:

- Thrombocytopenia defined as platelet count less than $10^{5} / \mathrm{mm}^{3}$ (severe thrombocytopenia- $<50,000 / \mathrm{mm}^{3}$ )

- Abnormal coagulogram [international normalized ratio (INR) $>$ 1.5 or aPTT $>50$ seconds]

- Treatment with antiplatelets (aspirin, clopidogrel, ticagrelor, etc.)

- Treatment with anticoagulants (enoxaparin, apixaban, warfarin, etc.), and

- Abnormal anatomy [(i) short neck, (ii) limited neck mobility (due to cervical spine fracture/fixation, neck contractures), (iii) neck infection/burns, (iv) redo tracheostomy or scar of previous tracheostomy, (v) class II obesity, (vi) USG neck showing thyroid abnormalities (goiter, aberrant thyroid nodule, very vascular thyroid), (vii) aberrant blood vessels near PDT site], (viii) USG neck showing deep seated trachea.

In the presence of hematological risk factors, the following precautions need to be observed-(i) information provided to blood bank about the procedure and reservation of blood products [packed red blood cells (PCV), fresh frozen plasma (FFP), and platelets] in the blood bank. The doses of antiplatelets or anticoagulants were not withheld.

Patients were divided into two groups-those at high risk of bleeding (having at least one risk factor for bleeding) and those without any risk factor for bleeding. The outcome of the patient (whether survived or died) in the ICU/hospital and number of days in ICU and in the hospital were collected from the hospital EMR.

\section{Data Analysis}

All these data were entered into a Microsoft ${ }^{\mathrm{TM}}$ Excel spreadsheet and analyzed. Statistical analysis was performed using SPSS version 19. Continuous variables were expressed as mean and standard deviation. Categorical variables were expressed as counts and percentages. Differences in the groups of patients were analyzed using the Chi-square test or Fischer's exact test. A $p$ value less than 0.05 was considered as statistically significant.

\section{Results}

One hundred and fifty-nine patients underwent PDT during the study period. The baseline characteristics of the patients are described in detail in Table 1. The mean age of the patients was 60.39 years, with the youngest patient being 21 years of age and the eldest being 104 years. Majority of the patients were male (65.41\%). The most common diagnoses for ICU admission were neurological diseases (43.39\%) involving the central and peripheral nervous systems and also included cerebrovascular accident (including both cerebral infarct and intracranial hemorrhage), head injuries/ traumatic brain injury, and Guillain-Barré syndrome. The other common diagnoses for ICU admission were sepsis (11.59\%) and myocardial infarction (6.92\%). Patients with trauma (polytrauma plus traumatic brain injury) comprised $4.4 \%$ of all patients. The most common indication for PDT was prolonged MV followed by 
Table 1: Patient characteristics of all patients who underwent percutaneous dilatational tracheostomy $(n=159)$

\begin{tabular}{|c|c|c|c|c|}
\hline Patient characteristics & All patients (\%) & $\begin{array}{l}\text { Patients at high risk of } \\
\text { bleeding, } n=87 \text { (\%) }\end{array}$ & $\begin{array}{l}\text { Patients with no risk factors } \\
\text { for bleeding, } n=72(\%)\end{array}$ & pvalue \\
\hline Age (years), mean (SD) & $60.39(19.75)$ & $65.45(18.7)$ & $55.32(19.18)$ & 0.347 \\
\hline Gender, (females) & $55(34.59)$ & $39(44.83)$ & $16(22.22)$ & 0.100 \\
\hline \multicolumn{5}{|l|}{ Diagnosis at ICU admission* } \\
\hline $\mathrm{ICH}$ & $39(24.53)$ & $10(11.49)$ & $29(40.28)$ & 0.182 \\
\hline CVA & $25(15.72)$ & $15(17.24)$ & $10(13.89)$ & 0.062 \\
\hline Head injury/traumatic brain injury & $4(2.51)$ & $0(0)$ & $4(5.56)$ & 0.025 \\
\hline GBS & $3(1.89)$ & $1(1.15)$ & $2(2.78)$ & 0.012 \\
\hline Sepsis & 19 (11.59) & $16(18.39)$ & $3(4.17)$ & 0.018 \\
\hline Myocardial infarction/cardiac arrest & $20(12.58)$ & $12(13.79)$ & $8(11.11)$ & 0.050 \\
\hline Polytrauma & $3(1.89)$ & $2(2.30)$ & $1(1.39)$ & 0.006 \\
\hline Others & $73(45.91)$ & $42(48.27)$ & $31(43.05)$ & 0.194 \\
\hline \multicolumn{5}{|l|}{ Indication for tracheostomy } \\
\hline Prolonged MV & $140(88.05 \%)$ & $74(85.05)$ & $66(91.67)$ & 0.415 \\
\hline To facilitate weaning & $4(2.52 \%)$ & $3(3.46)$ & $1(1.39)$ & 0.006 \\
\hline For airway protection & $14(8.80 \%)$ & $9(10.34)$ & $5(6.94)$ & 0.031 \\
\hline Pulmonary toilet & $1(0.63 \%)$ & $1(1.15)$ & $0(0.0)$ & 0 \\
\hline $\begin{array}{l}\text { Days to PDT insertion from day of admission, mean (SD) } \\
\text { (range) }\end{array}$ & $10.88(8.46)(1-63)$ & $11.02(8.36)(2-55)$ & $10.72(8.10)(1-63)$ & 0.067 \\
\hline $\begin{array}{l}\text { Days to PDT insertion from day of endotracheal } \\
\text { intubation, mean (SD) (range) }\end{array}$ & $10.49(7.713)(1-24)$ & $10.30(7.63)(1-44)$ & $10.72(8.10)(1-63)$ & 0.067 \\
\hline Patients surviving to ICU discharge & $112(70.44)$ & $60(68.96)$ & $52(72.22)$ & 0.327 \\
\hline Patients surviving to hospital discharge & $94(59.11)$ & $50(57.47)$ & $44(61.11)$ & 0.431 \\
\hline
\end{tabular}

*Some patients had more than one admitting diagnosis

$\mathrm{ICH}$, intracranial bleeding; CVA, cerebrovascular accident (except ICH); GBS, Guillain-Barré syndrome; MV, mechanical ventilation

Table 2: Risk factors in patients at high risk of bleeding $(n=87)$

\begin{tabular}{|c|c|c|}
\hline S. no. & Risk factors & $\begin{array}{l}\text { Number of patients* } \\
\text { (\%) }\end{array}$ \\
\hline \multicolumn{3}{|c|}{ (A) Hematological risk factors } \\
\hline 1 & Thrombocytopenia (platelet count $<10^{5} / \mathrm{mm}^{3}$ up to $50,000 / \mathrm{mm}^{3}$ ) & 25 \\
\hline 2 & Severe thrombocytopenia (platelet count $<50,000 / \mathrm{mm}^{3}$ ) & 4 \\
\hline 3 & Abnormal coagulogram (INR $>1.5$ or aPTT $>50$ seconds) & 2 \\
\hline 4 & Treatment with antiplatelet drugs (aspirin, clopidogrel, ticagrelor, etc.) & 27 \\
\hline 5 & Treatment with anticoagulant drugs (enoxaparin, apixaban, warfarin, etc.) & 17 \\
\hline \multicolumn{3}{|c|}{ (B) Anatomical risk factors } \\
\hline 1 & Short neck & 17 \\
\hline 2 & Limited neck mobility (due to cervical spine fracture/fixation, neck contractures) & 5 \\
\hline 3 & Neck infection/burns & 4 \\
\hline 4 & Redo tracheostomy or scar of previous tracheostomy & 3 \\
\hline 5 & Class II obesity & 4 \\
\hline 6 & USG neck showing thyroid abnormalities (goiter, aberrant thyroid nodule, very vascular thyroid, etc.) & 2 \\
\hline 7 & USG neck showing aberrant blood vessels near PDT site & 9 \\
\hline 8 & USG neck showing deep seated trachea & 6 \\
\hline
\end{tabular}

*Some patients had more than one risk factors

airway protection. The mortality rate in patients undergoing PDT was $29.66 \%$.

Eighty-seven (54.71\%) patients had risk factors for bleeding complications. The distribution and frequency of these risk factors is shown in Table 2. The most common hematological risk factors were thrombocytopenia (platelet count $<10^{5} / \mathrm{mm}^{3}$ upto $50,000 / \mathrm{mm}^{3}$ ) and treatment with antiplatelet drugs. The most common anatomical risk factors were short neck and USG neck showing aberrant blood vessels near PDT site. The distribution of risk factors in individual patients is shown in Table 3. Of the patients with risk factors for bleeding, almost two third of patients had a single risk factor, while one third had two risk factors. Three risk factors were present in very few patients.

The complications that occurred are shown in Table 4. Complication rate was $5.66 \%$. Transient hypoxia and hypotension were seen in seven $(4.40 \%)$ patients who responded almost 
Table 3: Patients at high risk of bleeding $(n=87)$ categorized according to the number of risk factors for bleeding in individual patient

\begin{tabular}{lll}
\hline S. no. & $\begin{array}{l}\text { Number of risk factors for } \\
\text { bleeding in one patient }\end{array}$ & Number of patients (\%) \\
\hline 1 & 1 risk factor & $53(60.92)$ \\
2 & 2 risk factors & $29(33.33)$ \\
3 & 3 risk factors & $5(5.75)$ \\
4 & $>3$ risk factors & 0 \\
\hline
\end{tabular}

Table 4: Occurrence of complications in patients who underwent percutaneous dilatational tracheostomy $(n=159)$

\begin{tabular}{|c|c|c|}
\hline S. no. & Complications & Frequency (\%) \\
\hline 1 & $\begin{array}{l}\text { Reduction of oxygen saturation below } 88 \% \\
\text { during the procedure (transient) }\end{array}$ & 4 \\
\hline 2 & Hypotension (responsive to fluids/inotropes) & 3 \\
\hline 3 & Cardiac arrest & 0 \\
\hline 4 & $\begin{array}{l}\text { Multiple }(>3) \text { attempts needed for tracheal } \\
\text { cannulation }\end{array}$ & 0 \\
\hline 5 & $\begin{array}{l}\text { Bleeding requiring more than three swabs } \\
\text { to be used and/or requiring surgical } \\
\text { intervention }\end{array}$ & 2 \\
\hline 6 & ETT cuff puncture & 0 \\
\hline 7 & $\begin{array}{l}\text { Fractured tracheal rings or posterior } \\
\text { tracheal wall was injured and visualized on } \\
\text { bronchoscopy }\end{array}$ & 0 \\
\hline 8 & Subcutaneous surgical emphysema & 0 \\
\hline 9 & Pneumothorax & 0 \\
\hline 10 & $\begin{array}{l}\text { Misplaced tracheostomy tube (false passage, } \\
\text { accidental extubation) }\end{array}$ & 0 \\
\hline 11 & PDT conversion to surgical tracheostomy & 0 \\
\hline
\end{tabular}

immediately to treatment. Bleeding was seen in only two patients at an incidence rate of $1.25 \%$. Bleeding occurred in one patient who did not have any risk factor for bleeding and in another patient who had hematological risk factors for bleeding (treatment with antiplatelets and anticoagulants). The bleeding was controlled with compression in the patients without risk factors and fresh frozen plasma and platelet transfusion in the patient with risk factors. None of the patients required blood or packed red blood cell transfusion. Other complications of PDT (pneumothorax, subcutaneous emphysema, ETT cuff puncture, fracture of tracheal rings, posterior tracheal wall injury, and misplaced tracheostomy tube) were not seen in any of the patients. None of the PDTs needed to be converted to ST.

\section{Discussion}

Percutaneous dilatational tracheostomy is a relatively safe procedure with a complication rate ranging from $1.6 \%$ to $14 \%$ as described in literature. ${ }^{15-18}$ The most common complication in large series of patients is bleeding. The other complications are quite infrequent. Fikkers et al. reported a rate of $1.4 \%$ for subcutaneous emphysema and $0.8 \%$ for pneumothorax. ${ }^{19}$ Dempsy et al. reported a large series of 576 PDTs and an early and late complication rate of $26 \%$, but most of the so-called complications were in fact technical difficulties, and the real rate of complications was 3\% early complications and $0.7 \%$ late complications (4.3\% had minor bleeding and only $1.04 \%$ had significant bleeding, $0.52 \%$ had surgical emphysema, and $0.17 \%$ had pneumothorax). In a study of 500 patients undergoing PDT with bronchoscopic guidance, Kost reported a complication rate of $6.5 \%$. The most common complications were oxygen desaturation and bleeding. No instances of pneumothorax or pneumomediastinum were reported.

Our complication (4.4\%) and bleeding rates were found to be comparable to these studies.

The low incidence of complications in our study may be due to the following factors: (i) routine use of USG neck prior to PDTs in all patients, so that there are no surprises during the procedures. (ii) Routine use of fiber optic bronchoscopy in all patients, for proper guidewire placement and to prevent tracheal ring and posterior tracheal damage. (iii) Experience of intensivists performing the PDTs ( $>50$ to $>100$ PDTs performed independently), so as to reduce surgical time and proper tissue handling. Kost also attributed the lack of pneumothorax or pneumomediastinum in their series to perform fiber optic bronchoscopy in all their patients while undertaking PDTs.

In patients with more than one risk factors for bleeding, there was some initial apprehension regarding bleeding. Biederlinden et al. in a study of 415 patients who underwent PDTs have found that coagulation abnormalities did not correlate well with acute (procedural) or chronic (bleeding continuing for $>24$ hours after PDT) bleeding in these patients. ${ }^{13}$ Further, heparin administration was not a risk factor for bleeding. They found that the risk of chronic bleeding correlated with prolonged aPTT $>50$ seconds and severe thrombocytopenia (platelet count of $<50,000 / \mathrm{mm}^{3}$ ). Also the presence of more than two coagulation abnormalities increased the risk of bleeding 10 times. They advocated the use of platelet transfusion in patients with severe thrombocytopenia prior to PDTs. Our results do not correlate with this. Although prolonged aPTT and severe thrombocytopenia were present in only six patients, excess bleeding was not seen in these patients. Thirty-four patients had multiple $(>1)$ risk factors for bleeding and bleeding was seen in one of these patients. The bleeding was controlled with treatment. However, to be on the safer side, when patients with multiple risk factors for bleeding undergo any surgical procedure appropriate care and precautions should be taken. These procedures should be undertaken with adequate blood bank backup and expertize to manage the bleeding complications, with surgical intervention, if needed..$^{13}$ As bleeding during the PDT procedure is usually due to injury to the blood vessels, the use of USG neck which helped us to locate aberrant vessels and visualize the neck vessels helped in preventing more episodes of bleeding.

A small study $(n=26)$ of patients on anticoagulants undergoing PDT reported an overall bleeding rate of $19 \%$, but most were minor bleeding (requiring on compression or dressing), with major bleeding (requiring blood transfusions or surgical intervention) occurring in two patients only.

Another study in 42 patients with severe thrombocytopenia undergoing PDT reported a bleeding rate of $5 \%$ (2 patients). ${ }^{8}$ However, they administered platelet transfusion prior to PDT. About half of their patients had additional coagulopathy in the form of raised INR ( $>1.5$ ) and prolonged aPTT ( $>40$ seconds). As the episodes of bleeding occurred in the patients with prolonged aPTT who were on heparin infusion, they advised temporary interruption of heparin infusion.

Our study has shown that it is safe to perform PDT in patients with severe thrombocytopenia, patients on antiplatelet and anticoagulation therapy, and patients with multiple risk factors 
for bleeding. There is no need for interruption of therapy or prior transfusion of platelets or plasma. The limitations of our study are that it is single-center study and hence the generalizability of our data to other centers may be limited. Also data were collected retrospectively. Hence, these findings should be confirmed in prospective trials. Although we have reported a large number of patients, our study may still be underpowered for detecting rare complications such as tracheoinnominate fistulas or PDT-related deaths. $^{20}$

\section{Conclusion}

Percutaneous dilatational tracheostomy is performed routinely in the ICU for patients on prolonged MV and for airway protection. It is a relatively safe procedure and can be performed even in the presence of relative contraindications such as thrombocytopenia, concurrent treatment with antiplatelet and anticoagulation drugs, and in the presence of abnormal local anatomy. Whenever risk factors for bleeding are present, PDT should be performed by experienced operator under bronchoscopic guidance with prior USG of the neck.

\section{References}

1. Freeman BD, Isabella K, Lin N, Buchman TG. A meta-analysis of prospective trials comparing percutaneous and surgical tracheostomy in critically ill patients. Chest 2000;118(5):1412-1418. DOI: 10.1378/chest.118.5.1412.

2. De Leyn P, Bedert L, Delcroix M, Depuydt P, Lauwers G, Sokolov Y, et al. Tracheotomy: clinical review and guidelines. Eur J Cardiothorac Surg 2007;32(3):412-421. DOI: 10.1016/j.ejcts.2007.05.018.

3. Delaney A, Bagshaw SM, Nalos M. Percutaneous dilatational tracheostomy vs surgical tracheostomy in critically ill patients: a systematic review and meta-analysis. Crit Care 2006;10(2):R55. DOI: 10.1186/cc4887.

4. Cheung NH, Napolitano LM. Tracheostomy: epidemiology, indications, timing, technique, and outcomes. Respir Care 2014;59(6):895-915. DOI: 10.4187/respcare.02971.

5. Madsen KR, Guldager H, Rewers M, Weber SO, Jacobsen KK, White J. Danish guidelines 2015 for percutaneous dilatational tracheostomy in the intensive care unit. Dan Med J 2015;61(3):B5042.

6. Cabrini L, Landoni G, Greco M, Costagliola R, Monti G, Colombo S, et al. Single dilator vs. guide wire dilating forceps tracheostomy: a metaanalysis of randomised trials. Acta Anaesthesiol Scand 2014;58(2):135-142. DOI: 10.1111/aas.12213.

7. Sanabria A. Which percutaneous tracheostomy method is better? A systematic review. Resp Care 2014;59(11):1660-1670. DOI: 10.4187/ respcare.03050.
8. Kluge S, Meyer A, Kuhnelt P, Baumann HJ, Kreymann G. Percutaneous tracheostomy is safe in patients with severe thrombocytopenia. Chest 2004;126(2):547-551. DOI: 10.1378/chest.126.2.547.

9. Beiderlinden M, Groeben H, Peters J. Safety of percutaneous dilational tracheostomy in patients ventilated with high positive endexpiratory pressure (PEEP). Intensive Care Med 2003;29(6):944-948. DOI: 10.1007/s00134-003-1656-8.

10. Mallick A, Bodenham AR. Tracheostomy in critically ill patients. Eur J Anaesthesiol 2010;27(8):676-682. DOI: 10.1097/ EJA.0b013e32833b1ba0.

11. Guinot PG, Zogheib E, Petiot S, Marienne J-P, Guerin A-M, Monet P, et al. Ultrasound-guided percutaneous tracheostomy in critically ill obese patients. Crit Care 2012;16(2):R40. DOI: 10.1186/cc11233.

12. Rajajee V, Fletcher J, Sheehan K, Jacobs T. Real time ultrasound reduces complications of percutaneous tracheostomy. Crit Care Med 2013; 41.

13. Beiderlinden M, Eikermann M, Lehmann N, Adamzik M, Peters J. Risk factors associated with bleeding during and after percutaneous dilational tracheostomy. Anaesthesia 2007;62(4):342-346. DOI: 10.1111/j.1365-2044.2007.04979.x.

14. Stehling LC, Doherty DC, Faust RJ, Greenburg AG, Harrison CR, Landers DF, et al. Practice guidelines for blood component therapy: a report by the American Society of Anesthesiologists Task Force on Blood Component Therapy. Anesthesiology 1996;84(3):732-747. DOI: 10.1097/00000542-199603000-00032.

15. Dempsey GA, Grant CA, Jones TM. Percutaneous tracheostomy: a 6 year prospective evaluation of the single tapered dilator technique. Br J Anaesth 2010;105(6):782-788. DOI: 10.1093/bja/aeq238.

16. Fikkers BG, Briede IS, Verwiel JMM, van den Hoogen FJA. Percutaneous tracheostomy with the Blue RhinoTM technique: presentation of 100 consecutive patients. Anaesthesia 2002;57(11):1094-1097. DOI: 10.1046/j.1365-2044.2002.02834.x.

17. Diaz-Reganon G, Minambres E, Ruiz A, Gonzalez-Herrera S, Holanda-Pena M, Lopez-Espadas F. Safety and complications of percutaneous tracheostomy in a cohort of 800 mixed ICU patients. Anaesthesia 2008;63(11):1198-1203. DOI: 10.1111/j.1365-2044.2008. 05606.x.

18. Kost KM. Endoscopic percutaneous dilatational tracheostomy: a prospective evaluation of 500 consecutive cases. Laryngoscope 2005;115(10 Pt 2):1-30. DOI: 10.1097/01.MLG.0000163744. 89688.E8.

19. Fikkers BG, van Veen JA, Kooloos JG, Pickkers P, van den Hoogen FJA Hillen $B$, et al. Emphysema and pneumothorax after percutaneous tracheostomy: case reports and an anatomic study. Chest 2004;125(5):1805-1814. DOI: 10.1378/chest.125.5.1805.

20. Simon M, Metschke M, Braune SA, Püschel K, Kluge S. Death after percutaneous dilatational tracheostomy: a systematic review and analysis of risk factors. Crit Care 2013;17(5):R258. DOI: 10.1186/ cc13085. 